\title{
PWE-054 THE VALUE OF FAECAL CALPROTECTIN IN THE INVESTIGATION OF SUSPECTED EARLY-ONSET INFLAMMATORY BOWEL DISEASE
}

doi:10.1136/gut.2011.239301.317

S J Lawrence, ${ }^{1}$ P Henderson, ${ }^{1,2,}{ }^{*}$ A Casey, ${ }^{1}$ K Kingstone, ${ }^{3}$ P Rogers, ${ }^{1}$ P M Gillett, ${ }^{1}$ D C Wilson ${ }^{1,2}{ }^{1}$ Department of Paediatric Gastroenterology, Hepatology and Nutrition, Royal Hospital for Sick Children, Edinburgh, UK; ${ }^{2}$ Department of Child Life and Health, University of Edinburgh, Edinburgh, UK; ${ }^{3}$ Department of Clinical Chemistry, Western General Hospital, Edinburgh, UK

Introduction The measurement of faecal calprotectin (FC) in patients with suspected bowel inflammation has become routine in many centres in recent years. Although FC levels have been shown to correspond with radio-nucleotide labelled neutrophil scans and endoscopic appearances in those with inflammatory bowel disease (IBD), it is still unclear as to the value of FC to inform IBD diagnosis, especially with regard to endoscopic investigation.

Methods The records of the cohort of all IBD patients who were diagnosed (using standard endoscopic assessment) within 
the Department of Paediatric Gastroenterology in Edinburgh between 1 January 2005 and 30 June 2010 (aged 1-17 years), with a FC performed during initial workup were identified. The local laboratory results system and endoscopy lists were reviewed and a matched non-IBD control who had similarly undergone endoscopy and also had a referral FC level available was identified. Only FC values taken at referral or initial investigation (not repeat samples) in those without any previous gastroenterological diagnosis were included. Patients who provided insufficient samples $(<2 \mathrm{~g})$ and those less than 1 year of age were excluded.

Results A total of 218 patient samples met the inclusion criteria. These included samples from patients with IBD $(n=109)$ (63 Crohn's disease, 22 ulcerative colitis, 24 IBD-U) and 109 controls. The IBD and control group consisted of 49 females: 60 males and 41 females: 67 males respectively $(p=0.364)$. In the IBD group 103/109 (94\%) had a FC level $>50 \mu \mathrm{g} / \mathrm{g}$ compared to $49 / 109(45 \%)$ in the control group $(p=<0.0001)$. However, the number of controls with values greater than $>200 \mu \mathrm{g} / \mathrm{g}$ was significantly lower - 19/109 (17\%) compared to 99/109 (91\%) for IBD $(\mathrm{p}=<0.0001)$. Using the standard laboratory lower reference range of $<50 \mu \mathrm{g} / \mathrm{g}$ the sensitivity of FC for IBD diagnosis was $94 \%$ (95\% CI 88 to 98), specificity 55\% (95\% CI 45 to 65) with a PPV of $68 \%$ (95\% CI 60 to 75$)$ and NPV of 91\% (95\% CI 81 to 97$)$. However, using the locally accepted clinical normal range of $<200 \mu \mathrm{g} / \mathrm{g}$ the specificity increased dramatically to $83 \%$ (95\% CI 74 to 89 ) with sensitivity 91\% (95\% CI 84 to 96), PPV $84 \%$ (95\% CI 76 to 90) and NPV $90 \%$ (95\% CI 82 to 95).

Conclusion It can be seen that FC is a useful tool in determining those who may require endoscopy for suspected bowel inflammation. Those with IBD have significantly higher FC levels at referral and therefore elevated values $>200 \mu \mathrm{g} / \mathrm{g}$ should prompt further GI investigation.

Competing interests None.

Keywords Faecal calprotectin, Inflammatory Bowel Disease, Paediatrics. 